\title{
S-MATRICES FOR PLANCKIAN SCATTERING
}

\author{
Sebastian de Haro \\ Spinoza Insitute, Utrecht University \\ Leuvenlaan 4, 3584 CE Utrecht \\ and \\ Institute for Theoretical Physics \\ Utrecht Univerisity, Princetonplein 5 \\ 3584 CC Utrecht, The Netherlands \\ haro@phys.uu.nl
}

String theory seems to give a unitary description of physics in the neighbourhood of certain black holes. A nice example of this is the AdS/CFT conjecture, where processes in the near-horizon region of several black branes are described by a conformal field theory which is unitary. However, it is hard to understand from the CFT where exactly Hawking's argument goes wrong.

An alternative but related program for understanding these issues was started by 't Hooft, who considered the near-horizon region of the Schwarzschild black hole, namely flat space. The interactions between outgoing Hawking radiation and ingoing particles, which near the black hole are boosted to the speed of light, can then be simulated by the gravitational interactions between massless particles. An $S$-matrix for their scattering can then readily be computed. For a review on the $S$-matrix Ansatz we further refer to [1].

In this note we discuss the quantum mechanical properties of this model, and its possible relation to the AdS/CFT conjecture.

\section{THE FLAT-SPACE S-MATRIX}

To understand how massless particles scatter gravitationally, one must first obtain them as a solution of the gravity theory. Aichelburg and Sexl [2] (see also [3]) found the following metric for a massless particle in flat space:

$$
\mathrm{d} s^{2}=\mathrm{d} u \mathrm{~d} v-p_{v} f(\tilde{x}) \delta(v) \mathrm{d} v^{2}+\mathrm{d} \tilde{x}^{2} .
$$


Now one would like to compute how test particles interact with the shockwave (1.1). Notice that trajectories of test particles that cross the shockwave are shifted by an amount $p_{v} f$. Indeed, the computation of geodesics in the metric (1.1) gives:

$$
u=\theta(v) p_{v} f(\tilde{x}) .
$$

From (1.2), the S-matrix for the scattering process can be computed [1]. It has a very simple form, since the interaction is described by a phase factor on the wave function. The amplitude is then

$$
\mathcal{A}=\left\langle p_{1}^{\text {out }} \cdots p_{M}^{\text {out }} \mid p_{1}^{\text {in }} \cdots p_{N}^{\text {in }}\right\rangle \int=\prod_{i j} \mathrm{~d} \tilde{x}_{i} \mathrm{~d} y_{j}\left|x_{i}-y_{j}\right|^{i G_{\mathrm{N}} p_{i}^{\text {in }} p_{j}^{\text {out }}}
$$

This is the well-known Veneziano amplitude, with an imaginary tension which is related to Newton's constant.

\section{QUANTUM MECHANICS OF SHOCK-WAVES}

The amplitude (1.3) suggests some sort of relation to string theories. It is therefore tempting to study how particles interacting through (1.1) behave as first quantised quantum mechanical systems. It turns out that the consistency of the operator algebra with the interactions imposes [4]:

$$
\begin{aligned}
{\left[X^{\mu}(\tilde{\sigma}), P^{\nu}\left(\tilde{\sigma}^{\prime}\right)\right] } & =i\left(g^{\mu \nu}+B^{\mu \nu}\right) \delta\left(\tilde{\sigma}-\tilde{\sigma}^{\prime}\right) \\
{\left[X^{\mu}(\tilde{\sigma}), X^{\nu}\left(\tilde{\sigma}^{\prime}\right)\right] } & =i G_{\mathrm{N}} \epsilon^{i j} \partial_{i} X \partial_{j} X \epsilon^{\mu \nu \alpha \beta} f\left(\tilde{\sigma}, \tilde{\sigma}^{\prime}\right),
\end{aligned}
$$

where $B^{\mu \nu}$ is a certain combination of $X, P$, and their derivatives, and $\tilde{\sigma}$ are the coordinates on the shockwave. This implies a generalisation of Heisenberg's principle,

$$
\begin{aligned}
\Delta x \Delta y & \geq \mathcal{O}\left(\ell_{\mathrm{Pl}}^{2}\right) \\
\Delta x \Delta p & \geq \frac{\hbar}{2}+\mathcal{O}\left(\ell_{\mathrm{Pl}}^{2} p_{\mathrm{in}} / b\right),
\end{aligned}
$$

where $b$ is the distance between the particles in transverse space. Notice that these are relations between operators corresponding to different particles.

It is at a first glance not clear why $X$ and $P$, when regarded as operators acting on Hilbert space, have a commutation relation different from the usual one. The explanation is as follows. Due to interactions, momentum transfer between the particles forces the canonical operator to be redefined according to the momentum exchange. One indeed finds [4] that there is an operator,

$$
\Pi^{\mu}=\left(g^{\mu \nu}-B^{\mu \nu}\right) P_{\nu},
$$


which has a canonical commutation relation with the position of the particle. One can verify that, classically, equation (1.8) gives the momentum exchange with the shockwave, which can be checked by simple kinematics.

Let us now comment on the covariant form of (1.4) and (1.5). The original computation assumed the eikonal approximation, where the transverse momentum transfer between the particles was neglected. However, as it turns out, equations (1.4), (1.5) and (1.8) automatically include corrections to the next order ${ }^{1}$, which are fixed by covariance and consistency of the quantum algebra. That is the reason that (1.8) gives us not only the right expressions for the longitudinal momenta, but also for the transverse momenta. Integrating the momentum over the whole plane of the shockwave, one finds

$$
P_{\text {in }}^{\mu}=P_{\text {out }}^{\mu},
$$

which is nothing else than conservation of momentum during the collision. However, when regarded as a relation between operators in the inand out-Hilbert space, it means that the two Hilbert spaces are identical.

A feature of this simple model for the black hole is that one gets a unitary S-matrix. As stated by (1.9), it is enough to know how the out-operators act on states to know the Hilbert space structure of the ingoing particles. In this sense the theory is holographic, since one only needs to make measurements on the horizon of the black hole (namely, one needs to know how its shape changes due to the presence of infalling and outcoming particles) to have all information about the inside.

\section{THE $S$-MATRIX AND THE ADS/CFT CONJECTURE}

The content of this section is at present still work in progress [5].

It would be interesting to see what implications the S-matrix Ansatz has when applied to the AdS/CFT conjecture.

To develop this program, one first needs a description of massless particles in AdS, including their interactions. In [6], a solution analogous to (1.1) was found for AdS. It was further argued that shock-waves in AdS correspond to light-cone states in the CFT, states whose stressenergy tensor is a delta-function centered on the light cone. Work is done in order to prove this by direct computation on the AdS side.

The solution found by Horowitz and Itzhaki can be used to compute the S-matrix for massless particle scattering in AdS. When dealing with

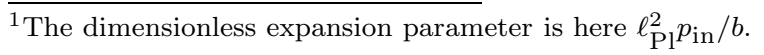


AdS, one has to be careful since in general an S-matrix has no meaning due to the presence of the boundary. Information can be lost or gained from the AdS boundary, and therefore the theory is not unitary. However, it turns out that for fields with special (reflective) boundary conditions, the S-matrix makes sense and can be computed. One gets a result which in the limit for large AdS radius reduces to (1.3), the flat-space S-matrix. Since the modes involved in this S-matrix are normalisable, from the CFT side the phase factor of the field corresponds to an interpolation between two different vacua of the theory on both sides of the light-cone. This will be reported elsewhere [5].

If one had an equation like (1.5) in AdS, it would be very interesting to understand what implications this has for the CFT on the boundary.

\section{Acknowledgments}

This work has partly been done in collaboration with G. Arcioni and K. Skenderis. We are also very much benefitted from discussions with G. 't Hooft and S. Solodukhin.

\section{References}

[1] 't Hooft, G. (1996) The Scattering Matrix Approach for the Quantum Black Hole: an Overview, Int. J. Mod. Phys., A 11, pp. 46234688

[2] Aichelburg, P.C. and Sexl, R.U. (1971) On the Gravitational Field of a Massless Particle, Gen. Rel. and Grav., 2, pp. 303-312

[3] Dray, T. and 't Hooft, G.(1985) The Gravitational Shock Wave of a Massless Particle, Nucl. Phys., B253, pp. 173-188

[4] de Haro, S. (1998) Planckian Scattering and Black Holes, JHEP, 9810, 023

[5] G. Arcioni and S. de Haro, to appear S. de Haro and K. Skenderis, work in progress

[6] Horowitz, G. and Itzhaki, N. (1999) Black holes, shock waves, and causality in the AdS/CFT correspondence, JHEP, 9902, 010 